\title{
RESÍDUOS E LIXIVIAÇÃO DO HERBICIDA PICLORAM EM ÁGUA, EM ÁREA DE CANA-DE-AÇÚCAR
}

\author{
Antônio Luiz Cerdeira ${ }^{1}$, Maria Conceição P. Y. Pessoa ${ }^{2}$, \\ Pierina Sueli Bonato ${ }^{3}$, Regina Helena C. Queiroz ${ }^{4}$ e Vera Lucia Lanchote ${ }^{4}$
}

\author{
'Engo Agrônomo, Ph.D., Pesquisador. Embrapa Meio Ambiente. Caixa Postal 69. Jaguariúna, SP 13820-000 \\ cerdeira@cnpma.embrapa.br \\ ${ }^{2}$ Matemática, Ph.D., Pesquisadora. Embrapa Meio Ambiente. \\ ${ }^{3}$ Farmacêutica, Ph.D., Professora. Departamento de Física e Química, Faculdade de Ciências Farmacêuticas, Universidade de São \\ Paulo. Ribeirão Preto, SP 14040-903 \\ ${ }^{4}$ Farmacêutica, Ph.D., Professora. Departamento de Análises Clínicas, Toxicológicas e Bromatológicas, Faculdade de Ciências Farma \\ cêuticas, Universidade de São Paulo.
}

\section{RESUMO}

A região de Ribeirão Preto, SP, Brasil, é abastecida com água proveniente de mananciais subterrâneos advindos do aqüífero Guarani, importante lençol de água subterrânea da região centro-sul do país. Na microbacia do Córrego do Espraiado, localizado nesta região, encontra-se um dos pontos de recarga desse aqüífero. Nesse local, com predomínio da cultura de cana-de-açúcar e presença de solo arenoso, a água se infiltra para camadas mais profundas do solo podendo lixiviar defensivos aplicados na superfície, tornando-a vulnerável a essa exposição. O herbicida picloram é um dos produtos aplicados na cultura e para tal foi desenvolvido um método de análise de resíduo do herbicida para amostras de água coletadas na microbacia no período de 1996 a 2001. A avaliação da tendência do produto alcançar camadas mais profundas do solo foi efetuada por análise cromatográfica e também por simulação de sistemas (CMLS-94-"Chemical Movement in Layered Soils") para melhor entender o comportamento do herbicida. O método analítico utilizado em cromatografia gasosa permitiu correlação de $98,0 \%$ entre a área dos picos e a concentração existente, indicando que foi altamente eficiente. Não foi encontrado resíduo de picloram em água, nos anos de 1996 a 2001. Estudos com o modelo matemático indicaram as profundidades máximas finais registrados para o terceiro ano de 8,2 m para Areia Quartzosa cinza (AQ-Cin), 7,3 m para a Areia Quartzosa rosa (AQ-Ros) e 6,7 m para Areia Quartzosa amarela (AQ-Ama), seguidas pelos Latossolos Vermelho-Escuro ( LE-Col, 5,7 m) e Roxo (LRCol, 5,3 m) indicando possibilidades de lixiviação do picloram

Palavras-chave: comportamento, contaminação.

\section{ABSTRACT \\ Picloram residues and leaching in water in a sugar cane area}

The region of Ribeirão Preto, SP, Brazil, is located on the Guarani aquifer, the most important source of ground water in the South Central region of the country. The recharge area of this aquifer is located in the Espraiado watershed. The presence of sandy soil and a sugar cane crop treated with pesticides makes the aquifer vulnerable to groundwater contamination. The herbicide picloram was among the pesticides used. To study its behavior, a gas chromatography analytical method for residue analysis was developed and picloram was measured during the years of 1996 until 1999. The movement also was 
evaluated with simulation using the software CMLS-94-"Chemical Movement in Layered Soils". The gas chromatography analytical method resulted in a $98.0 \%$ correlation of the area in the graphics and the herbicide concentration, and indicated that it was highly effective. No residue of picloram was detected in water in any year from 1986 to 1989 . The simulation model CMLS have indicated the leaching down to maximum depth of $8.2 \mathrm{~m}$ for "Areia Quartzosa cinza" (AQ-Cin) sandy soil, $7.3 \mathrm{~m}$ for "Areia Quartzosa rosa" (AQ-Ros) sandy soil and 6.7m for "Areia Quartzosa amarela" (AQ-Ama) sandy soil, followed by latossols "Latossolos Vermelho-Escuro" ( LE-Col, $5.7 \mathrm{~m}$ ) and "Latossolos Vermelho-Roxo" (LR-Col, $5.3 \mathrm{~m}$ ), indicating possibilities of picloram leaching.

Key words: Behavior, contamination, herbicide.

\section{INTRODUÇÃO}

A região de Ribeirão Preto, SP, Brasil, é abastecida com água proveniente de mananciais subterrâneos advindos do aqüífero Guarani, o maior e mais importante lençol de água subterrânea de toda a região centro-sul do país. A extensão desse aqüífero ultrapassa fronteiras estaduais e internacionais, dado que o mesmo passa por oito estados brasileiros e partes da Argentina, Uruguai e Paraguai, ocupando uma área aproximada de $1200000 \mathrm{~km}^{2}$ (Cerdeira et al., 1988). $\mathrm{Na}$ microbacia do Córrego do Espraiado, localizado nesta região, encontra-se um dos pontos de recarga do aqüífero, por onde a água se infiltra para camadas mais profundas do solo. Diante do predomínio da monocultura intensiva de canade-açúcar (Saccharum officinarum L.), de herbicidas aplicados e da presença de solo arenoso, aumenta-se a vulnerabilidade da água subterrânea à exposição a esses produtos aplicados na superfície.

Entre os produtos aplicados na cultura cita-se o uso do herbicida picloram (ácido 4-amino-3,5,6-tricloro-2piridinacarboxílico), com relatos de presença em água (Pang at al., 2000). Trata-se de um herbicida de ação hormonal e sistêmica em plantas, que mata ou afeta o crescimento das mesmas interferindo no metabolismo de nitrogênio. Picloram tem persistência moderadamente alta no ambiente com meia vida estimada em 20 a 300 dias, com média de 90 dias (Wauchope et al., 1992). Fotodegradação é importante apenas na superfície do solo, sendo degradado principalmente por microorganismos aeróbicos (Weed Science Society of America, 1994)

Em ambientes aquáticos, a velocidade de degradação do herbicida é acelerada com o aumento de nutrientes, sedimento e carbono orgânico dissolvido. A meia-vida do picloram em água em estudos de laboratório é de 2,6 dias (Howard, 1991). Em lagoas, em fazendas, encontrou-se até $1 \mathrm{mg} / \mathrm{L}$ após pulverizações, dependendo também das condições de oxigenação da água (U.S. Department of Agriculture, 1984). Alguns herbicidas hormonais, como o 2,4-D, tem sido detectados em baixas concentrações nas águas superficiais do Canadá e de cinco estados americanos (Sánchez-Brunete et al., 1991).

Para prevenir problemas de contaminação de água com defensivos, os países da Comunidade Européia aceitam 0,1 $\mu \mathrm{g} / \mathrm{L}$ a concentração máxima admissível (MAC) de um com- posto individual na água potável, sem no entanto ultrapassar $0,5 \mu \mathrm{g} / \mathrm{L}$ quando considera-se a soma de todos. Limites semelhantes são adotados por outros países como Estados Unidos e Canadá (Carney, 1991, Walker et al., 2000). No Brasil, a portaria $n^{\circ} 1469$, de 29/12/2000 estabelece os padrões de qualidade para água e os herbicidas estão com valores máximos aceitáveis de duas partes por bilhão (ppb).

Atualmente, a técnica da cromatografia líquida de alta eficiência vem sendo empregada na análise de herbicidas que absorvem na região do ultravioleta e que apresentam alto coeficiente de extinção molar. O emprego de detetores por arranjo de diodos ou por ultravioleta tem capacidade de analisar defensivos em concentrações inferiores a $0,1 \mu \mathrm{g} / \mathrm{L}$ de água (Lee \& Chau, 1983). Vários outros métodos também estão sendo propostos, como o uso de ELISA (Walker et al., 2000), entre outras derivações (Vink \& Poll, 1996; Farran et al., 1999; Houben et al., 1999).

A avaliação de risco ambiental do uso de picloram na área do Córrego do Espraiado também pode ser avaliada utilizando-se técnicas de simulação. (Pessoa et al., 1999). Neste sentido, o CMLS-94-"Chemical Movement in Layered Soils" (Nofziger \& Hornsby, 1994), possibilita a avaliação de potencial, lixiviação e persistência de defensivos em solo. O simulador disponibiliza valores de profundidade e concentração do defensivo aplicado, fornecendo a opção de saída gráfica para o acompanhamento de profundidades máximas alcançadas e respectivas concentrações presentes no perfil do solo ao longo do tempo simulado.

\section{MATERIAL E MÉTODOS}

Após levantamento inicial de uso de herbicidas na área, identificou-se que o herbicida picloram, Dontor, $(26,7 \mathrm{~g} / \mathrm{L}$ de ingrediente ativo), classe toxicológica 1, foi aplicado comercialmente na microbacia, na área de cana de açúcar, aproximadamente $80 \%$, na dose de $0,1 \mathrm{~kg} / \mathrm{ha}$ de ingrediente ativo a partir de 1995, em pré-emergência. Foram coletadas amostras em novembro, em água superficial no final do Córrego Espraiado, onde o mesmo começa a se transformar em uma lagoa natural na microbacia, durante os anos agrícolas de 1996 a 1999 e de poço localizado na área, no ano 2001. As amostras foram analisadas segundo o procedimento proposto e confirmado para avaliar a contaminação por resíduo do 
herbicida picloram, empregando cromatografia gasosa com captura de eletrons (Waliszewski \& Szymczwski, 1985; Siltanen \& mutanen, 1985; Gurka et al., 1986) de acordo com o protocolo desenvolvido a seguir:

\section{Reagentes}

A solução-padrão de picloram (ácido 4-amino-3,5,6tricloro-2-piridinacarboxílico 99,6\% EPA) foi preparada em metanol grau cromatografia (Merck) na concentração de 19,92 $\mu \mathrm{g} / \mathrm{mL}$. A partir dessa solução estoque foram preparadas diluições nas concentrações de 1,594, 0,996 e 0,498 $\mu \mathrm{g} / \mathrm{mL}$. A solução do padrão interno, ácido 3,4 diclorobenzóico, foi preparada em metanol grau cromatografia (Merck) na concentração de $40,0 \mu \mathrm{g} / \mathrm{mL}$. A solução de hidróxido de sódio $6 \mathrm{M}$ empregada no procedimento de hidrólise foi preparada em água purificada no sistema MILLI Q ${ }^{\circledR}$ (Millipore). A solução metanólica de ácido clorídrico foi preparada em metanol secado $(0,01 \%$ de água, para P.A., Merck), na concentração de $0,5 \mathrm{~N}$. No procedimento de extração foi empregado o diclorometano grau análise de resíduo (Grupo Química), e na derivação foi empregado tolueno grau análise de resíduo (Grupo Química).

\section{Análise cromatográfica}

A análise do herbicida nas amostras de água foi realizada empregando o cromatógrafo a gás GC17-A equipado com injetor "split/splitless" e detector por captura de elétrons (Shimadzu). A separação do herbicida e padrão interno foi realizada empregando uma coluna capilar DB-5, 0,25 mm x $30,0 \mathrm{~m}$, espessura do filme de $0,25 \mu \mathrm{m}$ (J \& W Scientific), com a programação de temperatura iniciando em $180^{\circ} \mathrm{C}(5$ min) e finalizando em $255^{\circ} \mathrm{C}$, com aquecimento de $25^{\circ} \mathrm{C}$ / min. A temperatura do vaporizador e detector foi estabelecida em 220 e $225^{\circ} \mathrm{C}$, respectivamente. $\mathrm{O}$ gás de arraste empregado foi o nitrogênio, na vazão total de $22 \mathrm{~mL} / \mathrm{min}$. O injetor foi operado no modo "split", sendo que o volume injetado foi de $2 \mu \mathrm{L}$.

\section{Preparação de amostras e procedimento de hidrólise e extração}

As amostras de águas foram filtradas através de membranas de 0,45 $\mu \mathrm{m}$ (Millipore) para remoção de material em suspensão e, a seguir, alíquotas de $100 \mathrm{~mL}$ foram transferidas para funis de separação e alcalinizadas com $300 \mu \mathrm{L}$ da solução de $\mathrm{NaOH} 6 \mathrm{M}$. Após agitação durante 1 hora, foram adicionados $200 \mu \mathrm{L}$ de ácido sulfúrico (grau P.A., Merck) e 5 $\mathrm{mL}$ de diclorometano. A seguir, as misturas foram submetidas a agitação durante 20 min e as fases orgânicas foram recuperadas. As fases aquosas foram novamente extraídas com mais duas porções de $5 \mathrm{~mL}$ de diclorometano e os extratos foram combinados e evaporados a secura sob fluxo de ar. Os resíduos obtidos foram submetidos ao procedimento de derivação descrito a seguir.

\section{Procedimento de derivação}

Os resíduos obtidos na etapa de extração foram adicionados de $50 \mu \mathrm{L}$ da solução do padrão interno (ácido 3,4diclorobenzóico) e de $1 \mathrm{~mL}$ da solução de ácido clorídrico preparada em metanol secado na concentração de $0,5 \mathrm{~N}$. Após agitação em "mixer" durante $1 \mathrm{~min}$, os tubos foram aquecidos a $60^{\circ} \mathrm{C}$ (banho maria) durante 1 hora. Após resfriamento dos tubos em água corrente, os derivados obtidos foram adicionados de $2 \mathrm{~mL}$ de água e extraídos com $500 \mu \mathrm{L}$ de tolueno, com agitação mecânica durante 30 min. Após centrifugação, foram recuperados $100 \mu \mathrm{L}$ das fases orgânicas e $2 \mu \mathrm{L}$ foram cromatografados nas condições descritas anteriormente.

\section{Curvas de calibração}

As curvas de calibração foram construídas de maneira similar após enriquecimento de alíquotas de $100 \mathrm{~mL}$ de água purificada em sistema MILLI Q®, Millipore, com 100 $\mu \mathrm{L}$ cada uma das soluções-padrão do herbicida, resultando em concentrações de 0,498 a 1,594 $\mu \mathrm{g} / \mathrm{L}$ de picloram em água.

Simulação matemática de lixiviação de picloram em Latossolo Roxo (LR-Col), Latossolo Vermelho-Escuro (LECol), Areia Quartzosa Amarela (AQ-Ama), Areia Quartzosa Cinza (AQ-Cin) e Areia Quartzosa Rosa (AQ-Ros)

A simulação de picloram foi realizada com o objetivo de avaliar a tendência de contaminação de águas subterrâneas da área de afloramento do Aquífero Guarani, Córrego do Espraiado, pelo produto. Por meio dela avaliou-se a dinâmica do produto no perfil vertical de Latossolo Roxo, Latossolo Vermelho-Escuro, Areia Quartzosa Amarela, Areia Quartzosa Cinza e Areia Quartzosa Rosa dessa área de estudo.

A entrada de dados necessária para o uso do CMLS94 foi: a) cultura: Kc (coeficiente cultural) da cultura, profundidade de raiz da cultura, data de plantio; b) do defensivo: Koc (coeficiente de partição no carbono orgânico), t 1/2 (meia vida), profundidade de aplicação, data de aplicação; c) do solo (para cada horizonte): profundidade do horizonte, \% carbono orgânico, densidade do solo, conteúdo volumétrico de umidade (capacidade de campo, ponto de murcha permanente, saturação); d) climáticos: dados diários de temperaturas máxima e mínima, pluviosidade e demanda de evapotranspiração.

Os dados relativos às características dos solos simulados, Latossolo Roxo, Latossolo Vermelho-Escuro e Areia Quartzosa Cinza foram relatados por Pessoa et al.,1999). O $\mathrm{Koc}(\mathrm{L} / \mathrm{kg}$, coeficiente de adsorção na fase orgânica) foi calculado em função do Kd (coeficiente de adsorção) estimado 
por Freundlich e da \% CO (percentagem de carbono orgânico) nas diferentes profundidades de solo, utilizando-se a seguinte equação: $\mathrm{Koc}=(\mathrm{Kd} / \% \mathrm{CO}) * 100$, onde as percentagens de carbono orgânico por profundidades de solo foram reportados por Pessoa et al., 1988 e o valor de Kd (coeficiente de adsorção) utilizado foi de $16 \mathrm{~mL} / \mathrm{g}$, baseado na literatura internacional (Wauchope et al., 1992). A meia vida em solo do produto utilizada foi estimada em 90 dias, também baseado na literatura internacional (Wauchope et al., 1992). Não se tem informação técnica sobre esses valores disponibilizados na literatura nacional para condições do Brasil.

A aplicação simulada do produto deu-se em outubro do primeiro ano, um mês após a brotação de cana, na dose de $0,1 \mathrm{~kg} / \mathrm{ha}$. As simulações foram realizadas separadamente para os solos considerados, para um período de 3 anos consecutivos, com os dados climáticos de Ribeirão Preto, SP (Pessoa et al., 1988).

\section{RESULTADOS E DISCUSSÃO}

Inicialmente foram obtidos resultados no desenvolvimento de análise do herbicida picloram em água, empregando a cromatografia gasosa com coluna empacotada e detecção por captura de elétrons. Métodos citados na literatura faziam o uso de um novo procedimento de derivação baseado na esterificação dos ácidos carboxílicos empregando ácido clorídrico/metanol. Com a substituição da coluna empacotada por uma coluna capilar (DB-1301), o método foi ligeiramente alterado, possibilitando a análise simultânea de mais de um herbicida com o procedimento de preparação da amostra inicialmente proposto para o picloram. Quanto à curva de calibração, foi obtida, na análise do herbicida, relação linear entre as razões de área (área do herbicida/área do padrão interno) e as concentrações do herbicida na água (Fig. 1). O método analítico utilizado permitiu correlação de $98,0 \%$ entre a área dos picos e a concentração existente, indicando que foi altamente eficiente e foi obtida a completa resolução do herbicida e do padrão interno (Fig. 1).

No cromatograma obtido na análise de água enriquecida com o herbicida picloram e submetida ao procedimento de extração proposto, foi obtida a completa resolução do herbicida e do padrão interno, conforme mostrado no cromatograma (Fig. 2). Os resultados obtidos não demostraram quantidades detectáveis do herbicida nas amostras analisadas em nenhum dos anos de pesquisados, sendo os resultados todos semelhantes ao que está sendo apresentado para o primeiro ano (Fig. 2).

\section{Resultados Obtidos por Simulação.}

Pelos resultados obtidos por simulação percebe-se grande mobilidade inicial no perfil vertical do solo, atingindo profundidades de 3,0 $\mathrm{m}$ na metade do primeiro ano de

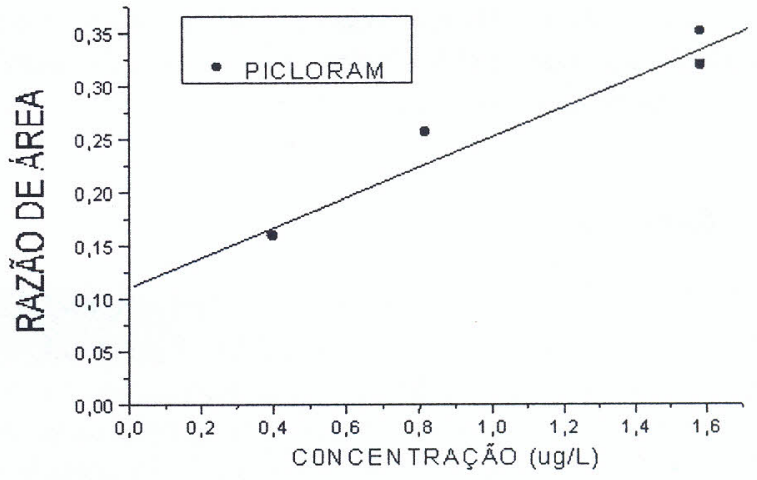

Figura 1- Curva de calibração referente a análise do herbicida picloram $(\mathrm{Y}=0,11+0,14 \mathrm{X} ; \mathrm{R}=0,980) \mathrm{em}$ água.

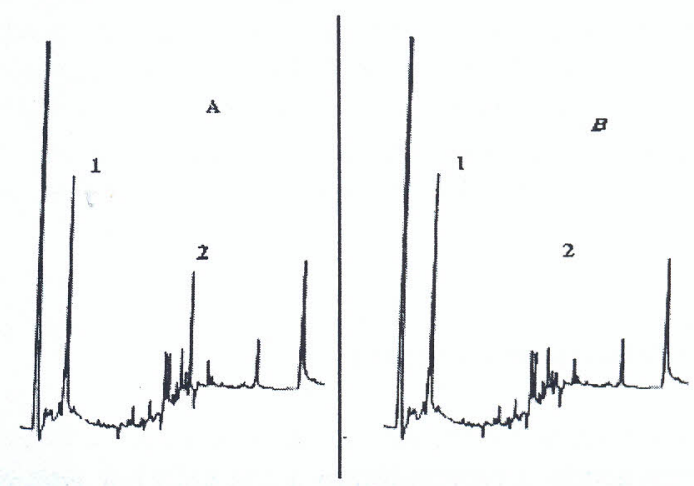

Figura 2- Cromatogramas obtidos na análise do herbicida picloram em água. $\mathbf{A}$ : pico $1=$ água enriquecida com padrão interno e pico $2=$ picloram. $\boldsymbol{B}$ : amostra de água coletada na bacia do espraiado. pico 2, picloram, não existente. Coluna $\mathrm{db}-50,25$ $\mathrm{mm}(0,25 \mathrm{~mm} \times 30 \mathrm{~m}) ; \mathrm{tv}=220^{\circ} \mathrm{c}$ durante 5 $\min ; 25^{\circ} \mathrm{c} / \mathrm{min}$ até $255^{\circ} \mathrm{c}$; td $=255^{\circ} \mathrm{c}$. fluxo nitrogênio $=22,0 \mathrm{ml} / \mathrm{min}$; injeção: split $=2 \mathrm{ml}$; detector = captura de elétrons.

aplicação do produto para todos os solos da área (Tabelas $1 \mathrm{e}$ 2). A profundidade alcançada no mesmo período de tempo é maior quando o produto é aplicado em Areia Quartzosa cin$\mathrm{za}$, onde o mesmo alcança profundidades superiores a $5 \mathrm{~m}$ (Tabela 1). Foi observado também que para as areias em geral, a profundidade registrada nesse mesmo período é superior a $4 \mathrm{~m}$ (Tabela 2 ).

Após essa rápida movimentação inicial de picloram para camadas mais profundas do solo, o produto sofre uma desaceleração na movimentação muito embora ela ainda persista até o final do período simulado.

Após o primeiro ano picloram apresenta maior mobilidade em areia quartzosa cinza $(5,18 \mathrm{~m})$, areia quartzosa rosa $(4,62 \mathrm{~m})$ e areia quartzosa amarela $(4,30 \mathrm{~m})$, sendo que os valores de profundidades obtidos para latossolos foram: 
Tabela 1. Profundidades (m) e quantidades de picloram (Qtd, $\mathrm{kg} / \mathrm{ha}$ ) resultantes nas simulações em latossolos comparados com areia quartzosa para três anos simulados consecutivamente. Ribeirão Preto, SP, 1999.

\begin{tabular}{ccccccc}
\hline \multicolumn{2}{c}{ Latossolo Roxo } & \multicolumn{2}{c}{ Areia Quartzosa Cinza } & \multicolumn{2}{c}{ Latossolo Vermelho-Escuro } \\
\hline & Prof(m) & Qtd(kg/ha) & Prof(m) & Qtd(kg/ha) & Prof(m) & Qtd(kg/ha) \\
\hline $1^{\circ}$ ano & 3,2 & $4,9 \times 10^{-3}$ & 5,2 & $4,9 \times 10^{-3}$ & 3,5 & $4,9 \times 10^{-3}$ \\
$2^{\circ}$ ano & 4,2 & $3,3 \times 10^{-4}$ & 6,4 & $3,3 \times 10^{-4}$ & 4,4 & $3,3 \times 10^{-4}$ \\
$3^{\circ}$ ano & 5,3 & $1,3 \times 10^{-4}$ & 8,2 & $1,3 \times 10^{-4}$ & 5,7 & $1,3 \times 10^{-4}$ \\
\hline
\end{tabular}

Tabela 2. Profundidades (m) e quantidades de picloram (Qtd, $\mathrm{kg} / \mathrm{ha}$ ) resultantes nas simulações em diferentes areia-quartzosas para três anos simulados consecutivamente. Ribeirão Preto, SP, 1999.

\begin{tabular}{ccccccc}
\hline & \multicolumn{2}{c}{ Areia Quartzosa Amarela } & \multicolumn{2}{c}{ Areia Quartzosa Cinza } & \multicolumn{2}{c}{ Areia Quartzosa Rosa } \\
\hline & Prof(m) & Qtd(kg/ha) & Prof(m) & Qtd(kg/ha) & Prof(m) & Qtd(kg/ha) \\
\hline $1^{\circ}$ ano & 4,3 & $4,9 \times 10^{-3}$ & 5,2 & $4,9 \times 10^{-3}$ & 4,6 & $4,9 \times 10^{-3}$ \\
$2^{0}$ ano & 5,2 & $3,3 \times 10^{-4}$ & 6,4 & $3,3 \times 10^{-4}$ & 5,6 & $3,3 \times 10^{-4}$ \\
$3^{\circ}$ ano & 6,7 & $1,3 \times 10^{-4}$ & 8,2 & $1,3 \times 10^{-4}$ & 7,3 & $1,3 \times 10^{-4}$ \\
\hline
\end{tabular}

Latossolo Roxo (3,2 m) e Latossolo Vermelho-Escuro (3,5 m) (Tabelas 1 e 2). Esta informação é importante para monitoramento da qualidade das águas dos lençóis sub-superficiais presentes na área de afloramento do Aqüífero, onde em metade da área de estudo encontram-se lençóis com níveis de profundidade de 0 a $5 \mathrm{~m}$ de profundidade (Pessoa et al., 1999). Parte da metade restante dessa área de estudo, encontram-se lençóis em níveis de profundidade de 5-10 m e na demais lençóis de 10-20 m e pequena área com lençóis de mais de $20 \mathrm{~m}$ (Pessoa et al., 1999). Para todos os solos, no primeiro ano a quantidade simulada do produto foi de $4,9 \times 10^{-3} \mathrm{~kg} / \mathrm{ha}$. Ressalta-se que não foi encontrado resíduo nas medições laboratoriais com cromatografia.

Ao final do segundo ano simulado, o produto atingiu maiores profundidades em Areia Quartzosa cinza (6,4 m), Areia Quartzosa rosa (5,6 m) e Areia Quartzosa amarela (5,2 $\mathrm{m})$, muito embora os valores das profundidades em Latossolo Vermelho-Escuro $(4,4 \mathrm{~m})$ e Latossolo Roxo $(4,2 \mathrm{~m})$ já se encontrem mais próximos daquele observado para Areia Quartzosa amarela. Os valores observados para as profundidades registradas, também indicam potencial de contaminação de água subterrânea por picloram em função dos níveis de lençóis já reportados. Nesse período, segundo ano, a quantidade do produto observada foi de $3,3 \times 10^{-4} \mathrm{~kg} / \mathrm{ha}$ (Tabela 2).

Os valores de profundidades finais registrados para $\mathrm{o}$ terceiro ano apontam a Areia Quartzosa cinza $(8,2 \mathrm{~m})$, Areia Quartzosa rosa (7,3 m) e Areia Quartzosa amarela $(6,7 \mathrm{~m})$, seguidas pelos Latossolos Vermelho-Escuro $(5,7 \mathrm{~m})$ e Roxo $(5,3 \mathrm{~m})$, que atingem os lençóis superficiais da área de afloramento, muito embora a quantidade presente de produ- to não seja significativa à saúde por encontrar-se na faixa de $1,3 \times 10^{-4} \mathrm{~kg} / \mathrm{ha}$. (Tabelas 1 e 2).

Assim, os resultados obtidos indicam que devido a maior mobilidade inicial de picloram, o mesmo deva ser monitorado para fins de contaminação de água subterrânea desde sua aplicação, quando as quantidades de produtos ainda são significativas durante o primeiro ano.

Os resultados corroboram com avaliações do índice de GUS (Gustafson, 1989) para o produto, que apresenta valor 5,5 indicando a existência de potencial de contaminação de águas subterrâneas pelo picloram (Pessoa et al., 2001). Também atende ao critério 1 do Método de GOSS (Goss, 1992), onde o picloram mostra ter potencial de transporte dissolvido em água (Pessoa et al., 2001). Ambos os métodos supra citados são utilizados pela EPA-Environmental Protection Agency dos EUA para selecionar e priorizar produtos a serem monitorados em campo.

\section{CONCLUSÕES}

Foi desenvolvido um método por cromatografia gasosa de análise de resíduo do herbicida picloram para amostras de água coletadas em área de cana-de-açúcar durante os anos de 1996 a 2001. Não foi encontrado resíduo de picloram em água.

O método cromatográfico analítico desenvolvido foi altamente eficiente.

Através de estudos com simulações, picloram apresentou maior potencial de contaminação de águas subterrâ- 
neas durante o primeiro ano, após a aplicação do produto. Nos solos arenosos o produto registrou maiores profundidades, sendo que os maiores valores foram obtidos para os solos arenosos quartzosos cinza. Ao final do terceiro ano, as profundidades registradas para picloram nesses solos poderia comprometer lençóis subterrâneos em níveis de profundidades de 0 a $5 \mathrm{~m}$, caso ainda houvesse resíduos nos solos, o que não é o caso.

\section{LITERATURA CITADA}

CARNEY, M. European drinking water standards. Journal AWWA, v. 83, p. 48-55, 1991.

CERDEIRA, A. L.; LANCHOTE, V. L.; QUEIROZ, R. C.; GOMES, M. A. F.; UETA, J. Resíduos de herbicidas em amostras de solo e água de área de cana-de-açúcar no Brasil. In: INTERNATIONAL CONFERENCE ON SUSTAINABLE AGRICULTURE IN TROPICAL AND SUBTROPICAL HIGHLANDS WITH ESPECIAL REFERENCE TO LATIN AMERICA, 1998, Rio de Janeiro. Anais... Rio de Janeiro: Embrapa Solos, 1998. CD-ROM

FARRAN, A.; SERRA, C.; SEPANIAK, M. J. Three different approaches for the separation of MCPA and 2,4-D by capillary electrophoresis. Journal of Chromatography A, v. 835, p. 209-215, 1999.

GOSS, D.W. Screening procedure for soils and pesticides for potential water quality impacts. Weed Technology, v. 6, p. 701-708, 1992.

GURKA, D. F.; SHORE, F. L.; PAN, S. T. Single-laboratory validation of EPA method 8150 for determination of chlorinated herbicides in hazardous waste. Journal of the Association of Official Analytical Chemists, v. 69, n. 6, p. 970-975, 1986.

GUSTAFSON, D. I. Groundwater ubiquity score: a simple method for assessing pesticide leachability. Environmental Toxicology and Chemistry, v. 8, n. 4, p. $339-357,1989$.

HOUBEN, A.; MEULENBERG, E.; NOIJ, T. ; GRONERT, C.; STOKS, P. Immuno affinity extraction of pesticides from surface water. Analytica Chimica Acta, v. 399, p. $69-74,1999$.

HOWARD, P. H. (Ed.). Handbook of environmental fate and exposure data for organic chemicals. v. 3 . Pesticides. Chelsea: Lewis Publishers, 1991. 101p.
LEE, H. B.; CHAU, A. S. Y. Analysis of pesticide residues by chemical derivation. VI. Analysis of ten acid herbicides in sediment. Journal of the Association of Official Analytical Chemists, v. 66, n. 4, p. 1023-1028, 1983.

NOFZIGER, D. L.; HORNSBY, A. G. CMLS-94-chemical movement in layered soils. Oklahoma: University of Florida - Department of Agronomy, 1994. 76 p.

PANG, L.; CLOSE, M. E.; WATT, J. P. C.; VINCENT, K. W. Simulation of picloram, atrazine, and simazine leaching through two New Zealand soils and into groundwater using HYDRUS-2D. Journal of Contaminant Hydrology, v. 44, n. 1, p. 19-46, 2000.

PARANHOS, S. B. Cana-de-açúcar: cultivo e utilização. Campinas: Fundação Cargill , 1987. 2v., 856 p.

PESSOA, M. C. P. Y.; GOMES, M. A. F.; DORNELAS DE SOUSA, M.; NICOLELLA, G.; CERDEIRA, A. L.; MONTICELLI, A . Simulação do movimento de herbicidas utilizados no monocultivo de cana-de-açúcar em Areia Quartzosa da área de recarga do Aquífero Guarani (antigo Botucatu) em Ribeirão Preto, SP. Revista Científica Rural, v. 3, n. 2, p. 11-19, 1988.

PESSOA, M. C. P. Y.; GOMES, M. A. F.; SOUZA, M. D. de; CERDEIRA, A. L.; NEVES, M. C.; NICOLELLA, G. Estudos de simulação do movimento vertical de herbicidas em solos com cana-de-açúcar na microbacia do córrego do Espraiado, Ribeirão Preto (SP). Jaguariúna: Embrapa Meio Ambiente, 1999. 44 p. (Embrapa Meio Ambiente. Boletim de Pesquisa, 1).

PESSOA, M. C. P. Y.; SCRAMIN, S.; FERRACINI, V. L.; CHAIM, A.; SILVA, A. de S.; CERDEIRA, A. L.; GOMES, M. A. F.; GUSSAKOV, K. C. Impacto ambiental do uso de agrotóxicos na qualidade das águas das bacias hidrográficas brasileiras - Estado da Arte. Jaguariúna: Embrapa Meio Ambiente; Brasília: Secretaria de Recursos Hídricos/Ministério do Meio Ambiente, 2001. 218 p. (Relatório Técnico de Consultoria para o SRH/MMA).

SÁNCHEZ-BRUNETE, C.; PÉREZ, S.; TADEO, J. L. Determination of phenoxy ester herbicides by gas and high-performance liquid chromatography. Journal of Chromatography, v. 522, p. 235-240, 1991.

SILTANEN, H.; MUTANEN, R. Formation of derivatives of chlorophenoxyacids and some other herbicides. Chromatographia, v. 20, n. 11, p. 685688,1985 . 
U.S. Department of Agriculture (U.S. Forest Service). Pesticide background statements. v.1. Herbicides. Washington, DC, 1984. p.10-17.

VINK, M.; POLL, J. M. Gas chromatographic determination of acid herbicides in surface water samples with electroncapture detection and mass spectrometric confirmation. Journal of Chromatography A, v. 733, p. 361-366, 1996.

WALISZEWSKI, S. M.; SZYMCZNSKI, G. A. Modified method for the GC determination of chlorophenoxyacids acetic herbicides (MCPA and 2,4-D) in soil and water. Fresenius Zeitschrift fur Analytische Chemie, v. 322, p. $510-511,1985$.
WALKER, A. E.; HOLMAN, R. E.; LEIDY, R. B. ELISA and GC/MS analysis of pesticide residues in North Carolina. Journal of the American Water Resources Association, v. 36, p. 67-74, 2000.

WAUCHOPE, R. D.; BUTTLER, T. M.; HORNSBY A. G.; AUGUSTIJN BECKERS, P. W. M.; BURT, J. P. SCS/ ARS/CES pesticide properties database for environmental decision making. Reviews of Environmental Contamination and Toxicology, v. 123, p. 1-157, 1992.

WEED SCIENCE SOCIETY OF AMERICA. Herbicide handbook. 7 ed. Champaign: WSSA, 1994. p.10-59. 